\title{
Una dinámica de larga duración para fomentar la participación en clases
}

\author{
A long term dynamic to encourage class participation \\ Ricardo Valdivia Pinto ${ }^{1 *} \quad$ Yordan Vera Castillo $^{2}$ \\ Recibido 27 de junio de 2018, aceptado 06 de agosto de 2018 \\ Received: June 27, 2018 Accepted: August 06, 2018
}

\begin{abstract}
RESUMEN
Este artículo presenta la descripción, implementación y evaluación de una dinámica de larga duración orientada a mejorar la participación de los estudiantes en clases. La dinámica está diseñada para llevarse a cabo durante la duración semestral de un curso, debido a lo cual es relevante el soporte tecnológico que mantenga el estado de la dinámica clase a clase.

La dinámica propuesta se basa en una variación de las dinámicas basadas en la votación sobre preguntas de selección múltiple, las que han sido ampliamente estudiadas y han dado origen a múltiples sistemas de soporte que permiten, entre otras ventajas: la reutilización de preguntas, la discusión de los resultados de la votación por parte de los estudiantes y la rápida retroalimentación al profesor. Sin embargo, también existen críticas, especialmente vinculadas a la complejidad en la elaboración de las preguntas y a su alcance en términos del aprendizaje. En contraste, esta dinámica propone el uso de preguntas abiertas para generar actividad en la clase y a lo largo de la duración del curso. La dinámica esta soportada por una plataforma que apoya al profesor en la selección del estudiante que responderá, de acuerdo a su grado de participación en la dinámica.
\end{abstract}

Esta dinámica y su soporte tecnológico fueron aplicados en un curso regular de Computación e Informática, produciendo resultados auspiciosos, en particular en el grado de satisfacción de los estudiantes.

Palabras clave: Aprendizaje activo, sistemas de respuestas en clases, preguntas abiertas.

\begin{abstract}
This paper presents the description, implementation, and evaluation of a long-term dynamic oriented to improve the participation of the students in classes. The dynamics are designed to be carried out during a semester course, due to which it is relevant the technological support that maintains the state of the dynamics class to class.

The proposed dynamics is based on a variation of voting-based dynamics on multiple-choice questions, which have been extensively studied and has given rise to multiple support systems that allow, among other advantages: reuse of questions, discussion of the voting results by students and rapid feedback to the teacher. However, there are also critical, especially linked to the complexity of developing questions and their scope regarding learning. In contrast, this dynamic proposes the use of open questions to generate activity in the class and throughout the course duration. The dynamics are supported by a platform that feeds the teacher in the selection of the student who will respond, according to their degree of participation in the dynamics.
\end{abstract}

\footnotetext{
1 EUIIIS. Universidad de Tarapacá. 18 de Septiembre 2222. Arica, Chile. E-mail: rvaldivi@uta.cl

2 EUIIIS. Universidad de Tarapacá. 18 de Septiembre 2222. Arica, Chile. E-mail: yordanveracastillo@gmail.com

* Autor de correspondencia.
} 
These dynamics and its technological support were applied in a regular course in Computer Science, producing auspicious results, particularly in the degree of student satisfaction.

Keywords: Active learning, classroom response systems, open questions.

\section{INTRODUCCIÓN}

El aprendizaje activo invita a los estudiantes a que se involucren en las actividades de la clase y no a que escuchen pasivamente una presentación [1]. Las técnicas de aprendizaje activo consideran la realización de actividades asociadas a la discusión y resolución de problemas. También, como parte de este proceso, los estudiantes deben discutir sobre el proceso de deducción que han utilizado.

Las técnicas de aprendizaje activo están basadas en el constructivismo, la teoría dominante en el área de la educación. En un ambiente educacional constructivo, los estudiantes construyen su propio conocimiento mientras trabajan como grupo a través de la interacción social, en un proceso reflexivo, guiado por el profesor [2].

El uso de sistemas basados en preguntas de selección múltiple (multiple choice questions, MCQ) ha sido ampliamente estudiado. Entre las ventajas del uso de MCQ [3] se indica que: estas proveen de rápida retroalimentación, pueden ser automáticamente evaluadas, y pueden almacenarse en bases de datos de preguntas para ser reutilizadas. Entre las desventajas, se argumenta que su construcción requiere de un esfuerzo considerable, que sólo pueden evaluar conocimiento y memorización, y no consideran aspectos de análisis y creatividad, provocando que los estudiantes adopten una actitud superficial al aprendizaje.

Uno de los primeros sistemas en soportar el uso de MCQ en clases soportadas por tecnología fue Classtalk [4], un sistema donde los estudiantes respondían MCQ utilizando dispositivos móviles. Su uso incluía la posibilidad de generar discusión con el resto de la clase, con posterioridad a la realización de la dinámica, utilizando histogramas que mostraban la distribución de las respuestas votadas por los estudiantes. Este es una variante de la dinámica de clases activas conocida como aprendizaje entre pares (peer instruction) [5].
Sobre él se señala que "el verdadero corazón del aprendizaje se produce cuando los estudiantes se involucran entre sí conversando sobre la base de las disonancias reveladas por la pantalla compartida" [6], lo que revela la importancia de provocar discusión en la clase.

Los estudios llevados a cabo en el uso de estos sistemas, conocidos genéricamente como sistemas de respuesta en clases (classroom response system, CRS) [7] o sistemas de comunicación en clases (classroom communication systems, CCS) [8], han mostrado que el rol de la tecnología es valioso al interior de la clase [6]. La tecnología provee anonimidad, rápida recolección de respuestas y la habilidad de producir una visualización compartida la cual mejora el reconocimiento de posiciones comunes. La tecnología entonces actúa como un catalizador, produciendo un cambio en el clima de la clase, la pedagogía y el aprendizaje resultante [9].

\section{DESCRIPCIÓN DE LA DINÁMICA}

Como una variante de los sistemas CRS, la dinámica descrita en este trabajo, considera un conjunto de características diferenciadoras orientadas a mejorar los problemas que involucra tanto la implementación como el uso de estas dinámicas. En particular, (a) se basa en el uso de preguntas abiertas, (b) el tiempo se extiende más allá de la clase, cubriendo el desarrollo de todo el semestre, y finalmente, (c) incorpora la evaluación como un factor de organización e incentivo en la dinámica.

El uso de preguntas abiertas pretende ampliar el espacio de preguntas posibles de realizar a los estudiantes, fortaleciendo tanto sus capacidades de reflexión y crítica, como sus habilidades comunicativas. Las preguntas realizadas clase a clase incentivan a los estudiantes para que participen activamente en la construcción de su conocimiento. La evaluación, basada en un proceso de acumulación de puntos: (a) permite que la dinámica proceda 
como un continuo (los estudiantes son informados de su rendimiento y esperan informados la próxima actividad), (b) incentiva la participación (los estudiantes desean optar a una mejor evaluación en cada clase), (c) requiere que el profesor organice la participación considerando las necesidades de cada estudiante.

Al comienzo del semestre, el profesor debe establecer una "meta" del curso basada en la cantidad de preguntas que debiese realizar, esto con el fin de optimizar las posibilidades que cada estudiante tendrá de participar de las actividades durante el transcurso del semestre. El sistema debe apoyar claramente esta característica, visualizando la participación y rendimiento de los estudiantes, ayudando al profesor a tomar mejores decisiones en cuanto a "que" estudiante seleccionar al momento de plantear una pregunta.
La Figura 1 describe por medio de una formalización en BPMN (Business Process Model and Notation) [10] la dinámica que es aplicada clases a clase. Adicionalmente, la Tabla 1 detalla cada una de las etapas involucradas en la misma.

\section{EL SISTEMA CRSOQ}

Para el soporte de la dinámica propuesta, se ha seguido la tendencia en el uso de sistemas web para el soporte de dinámicas CRS como Kahoot [11]. Estos sistemas permiten sustituir el original uso de tecleras (clickers) por la actual disponibilidad de dispositivos móviles (tablets o smartphones) como dispositivos de votación.

\section{Soporte de la Dinámica en la Clase}

Actualmente, el sistema desarrollado CRSoq (Classroom Response System Open Questions)

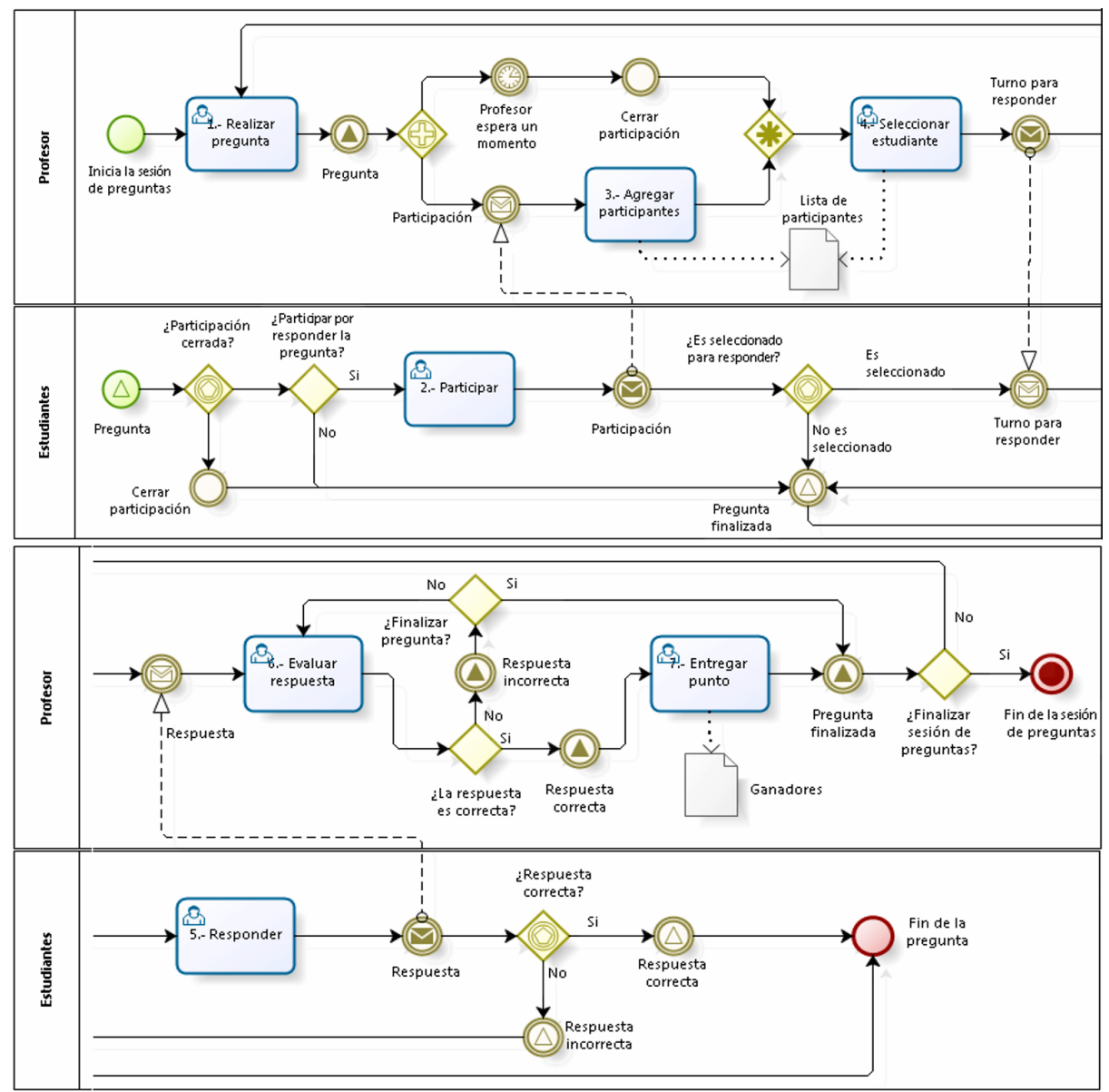

Figura 1. Diagrama BPMN de la Dinámica en clase. 
Tabla 1. Descripción de la dinámica.

\begin{tabular}{|c|c|c|}
\hline Actor & Evento & Descripción \\
\hline Profesor & & $\begin{array}{l}\text { El profesor realiza una pregunta a } \\
\text { los estudiantes. } \\
\text { (Aregunta }\end{array}$ \\
\hline Estudiante & & $\begin{array}{l}\text { Realizada la pregunta, se verifica si } \\
\text { la participación está cerrada. } \\
\text { (ㅁ) ¿Participación cerrada? }\end{array}$ \\
\hline Estudiante & & $\begin{array}{l}\text { Mientras no ocurra el evento } \\
\text { Cerrar participación, el } \\
\text { estudiante puede decidir participar. } \\
\text { iParticipar por responder la }\end{array}$ \\
\hline Estudiante & & $\begin{array}{l}\text { Si el estudiante decide participar, } \\
\text { envía un mensaje de participación } \\
\text { al profesor. Luego, debe esperar su } \\
\text { posible selección. } \\
\text { (D) ¿Es seleccionado para } \\
\text { responder? }\end{array}$ \\
\hline Estudiante & & $\begin{array}{l}\text { Si el estudiante decide no participar, } \\
\text { espera a que la pregunta finalice. } \\
\text { (4regunta finalizada }\end{array}$ \\
\hline Profesor & & $\begin{array}{l}\text { Una vez realizada la pregunta, } \\
\text { el profesor espera a que } \\
\text { los estudiantes decidan su } \\
\text { participación. Mientras se espera } \\
\text { la participación de los } \\
\text { estudiantes, se va actualizando la } \\
\text { lista de participantes. } \\
\text { Finalmente, el profesor procede a } \\
\text { cerrar la participación de los } \\
\text { estudiantes. }\end{array}$ \\
\hline Profesor & t) & $\begin{array}{l}\text { Una vez que se haya cerrado la } \\
\text { participación el profesor debe } \\
\text { seleccionar un estudiante para que } \\
\text { responda la pregunta, por lo que se } \\
\text { le avisa que es su turno para } \\
\text { responder. }\end{array}$ \\
\hline Estudiante & (1) & $\begin{array}{l}\text { ¿Es seleccionado para } \\
\text { responder? } \\
\text { Si el estudiante es seleccionado } \\
\text { debe ser notificado por el profesor } \\
\text { con su turno para responder. } \\
\text { El estudiante da su respuesta } \\
\text { a la clase. } \\
\text { Luego debe esperar el resultado de } \\
\text { la evaluación. } \\
\text { (1) ¿Respuesta correcta? }\end{array}$ \\
\hline
\end{tabular}

\begin{tabular}{|c|c|c|}
\hline Actor & Evento & Descripción \\
\hline Estudiante & & $\begin{array}{l}\text { En caso de que el estudiante no sea } \\
\text { seleccionado, debe esperar a que la } \\
\text { pregunta finalice. } \\
(\triangle \text { Pregunta finalizada. }\end{array}$ \\
\hline Profesor & & $\begin{array}{l}\text { El profesor determina si la respuesta } \\
\text { del estudiante es correcta. } \\
\text { ¿La respuesta es correcta? }\end{array}$ \\
\hline Profesor & & $\begin{array}{l}\text { Si la respuesta es incorrecta, } \\
\text { debe anunciarlo a la clase. } \\
\text { ¿Finalizar pregunta? } \\
\text { En caso de que decida continuar } \\
\text { con la pregunta, debe seleccionar } \\
\text { otro estudiante. } \\
\text { En caso de que decida finalizar la } \\
\text { pregunta, debe avisar a la clase que } \\
\text { la pregunta ha finalizado. }\end{array}$ \\
\hline Profesor & & $\begin{array}{l}\text { En caso de que la respuesta es } \\
\text { correcta, debe anunciarlo a la clase. }\end{array}$ \\
\hline Estudiante & & $\begin{array}{l}\text { Si la respuesta del estudiante es } \\
\text { incorrecta, el estudiante debe } \\
\text { esperar a que la pregunta finalice. } \\
\text { Pregunta finalizada }\end{array}$ \\
\hline Estudiante & & $\begin{array}{l}\text { Si la respuesta del estudiante es } \\
\text { correcta, finaliza la pregunta. } \\
\text { Fin de la pregunta }\end{array}$ \\
\hline Profesor & & $\begin{array}{l}\text { Una vez que se dio el aviso de que } \\
\text { la respuesta es correcta, el } \\
\text { profesor debe entregar el punto al } \\
\text { ganador, registrándolo en la lista de } \\
\text { ganadores. } \\
\text { Se avisa a la clase que la pregunta } \\
\text { ha finalizado iFinalizar sesión de } \\
\text { preguntas? }\end{array}$ \\
\hline Estudiante & (4) & $\begin{array}{l}\text { Una vez que la pregunta } \\
\text { ha finalizado, el resto de } \\
\text { los estudiantes concluye su } \\
\text { participación. } \\
\text { Fin de la pregunta }\end{array}$ \\
\hline Profesor & & $\begin{array}{l}\text { Si el profesor decide no finalizar la } \\
\text { sesión de preguntas, entonces debe } \\
\text { realizar otra pregunta y el proceso } \\
\text { vuelve a comenzar. }\end{array}$ \\
\hline Profesor & & $\begin{array}{l}\text { Si el profesor decide finalizar la } \\
\text { sesión de preguntas, se cierra la } \\
\text { dinámica. } \\
\text { Fin de la sesión de preguntas }\end{array}$ \\
\hline
\end{tabular}


incluye la gestión de cursos, estudiantes y base de preguntas de forma de soportar adecuadamente la dinámica en la clase. Se describirá este último aspecto:

\section{a. Inicio de la sesión de preguntas}

Al comenzar la actividad, el profesor puede elegir qué clase iniciar, haciendo click en el botón "play" (Figura 2).

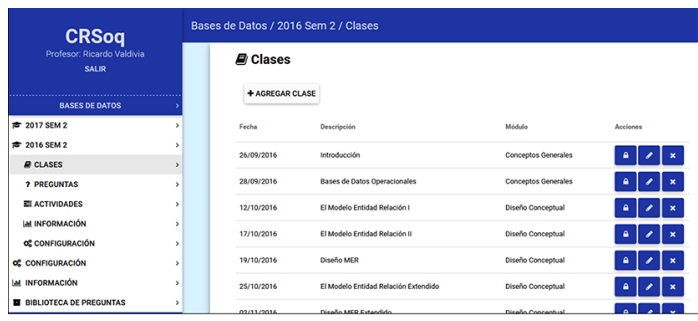

Figura 2. Inicio de la dinámica en la clase.

Una vez que el profesor inició la sesión de preguntas (Figura 3), puede elegir entre las preguntas que ya se encuentran ligadas a la clase, o crear preguntas en el momento.

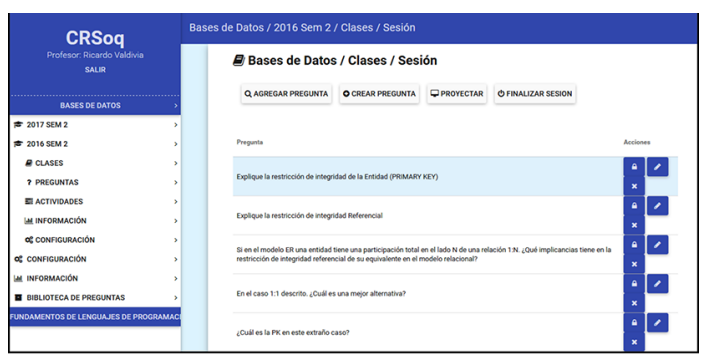

Figura 3. Inicio de la sesión de preguntas.

\section{b. Profesor realiza una pregunta}

Mientras el profesor decide que pregunta realizar, los estudiantes quedan en un estado de espera como se ve en la Figura 4. Esto forma parte de la primera

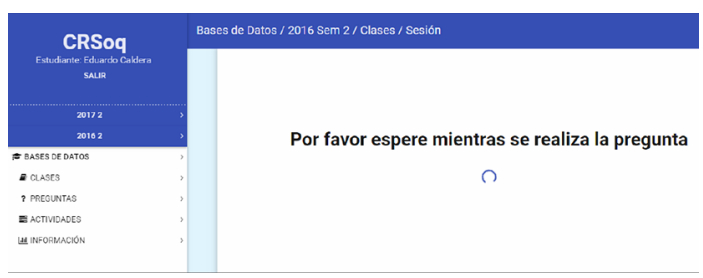

Figura 4. Espera por la pregunta. tarea "Realizar pregunta" de la descripción de la dinámica.

\section{c. Participación de los estudiantes}

Una vez que se realiza la pregunta a los estudiantes, se les habilita un botón, ver Figura 5. En el momento en que lo presionan, su participación se registra y es enviada al profesor. Esta etapa corresponde a la segunda tarea de la dinámica CRS.

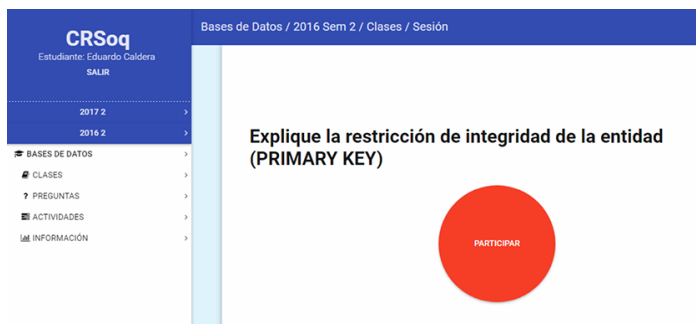

Figura 5. Botón de participación.

A medida que los estudiantes participan de la pregunta, el sistema se encarga de ejecutar la tercera tarea, la que consiste en agregar a los estudiantes a la lista de participantes. Esta lista se muestra en la vista del profesor, ver Figura 6. Cuando el profesor estima que ha pasado tiempo suficiente, cierra la participación.

\section{d. Selección del estudiante que debe responder} Luego de que el profesor ha cerrado la pregunta, sigue la cuarta tarea de la dinámica que consiste en la selección del estudiante que va a responder la pregunta. En este proceso, el sistema proporciona al profesor información basada en la participación de los estudiantes en la dinámica durante el semestre y la clase (sesión).

La Figura 6 muestra la información con la que cuenta el profesor para seleccionar al estudiante de la lista de participantes. Esta incluye el total de preguntas ya realizadas durante el semestre y la sesión, la cantidad de preguntas en las que han participado cada estudiante durante el semestre y la sesión, la cantidad de preguntas en las que no han participado cada estudiante durante el semestre y la sesión, la cantidad de preguntas que cada estudiante ha contestado correctamente durante el semestre y la sesión, la cantidad de preguntas que cada estudiante ha contestado incorrectamente durante el semestre 


\title{
Explique la restricción de integridad de la entidad (PRIMARY KEY)
}

\author{
UFINALIZAR PREGUNTA DETENER PARTICIPACIÓN
}

\# estudiante

\begin{tabular}{|c|c|c|c|c|c|c|c|}
\hline \multirow{3}{*}{1} & \multicolumn{7}{|c|}{ Manuel Tapia 19.356.766-5 } \\
\hline & Semestre & 94 & 14 & 80 & 5 & 2 & 7 \\
\hline & Sesión & 5 & 2 & 3 & 2 & 0 & 0 \\
\hline \multirow{4}{*}{2} & \multicolumn{7}{|c|}{ Alexander Escarate 19.492.869-6 } \\
\hline & & Total Preguntas & Participa & No Participa & Correctas & Incorrectas & No Seleccionado \\
\hline & Semestre & 94 & 55 & 39 & 11 & 5 & 39 \\
\hline & Sesión & 5 & 2 & 3 & 1 & 0 & 1 \\
\hline \multirow{4}{*}{3} & \multicolumn{7}{|c|}{ Eduardo Caldera 18.943.775-7 } \\
\hline & & Total Preguntas & Participa & No Participa & Correctas & Incorrectas & No Seleccionado \\
\hline & Semestre & 94 & 51 & 43 & 10 & 4 & 37 \\
\hline & Sesión & 5 & 4 & 1 & 1 & 0 & 3 \\
\hline
\end{tabular}

Figura 6. Lista de participantes.

y la sesión; y, finalmente, la cantidad de preguntas en las que no ha sido seleccionado cada estudiante durante el semestre y la sesión.

\section{e. Estudiante entrega su respuesta}

Una vez hecha la selección del estudiante, se le notifica su turno, ver Figura 7. El estudiante debe dar su respuesta al curso y al profesor, lo que corresponde a la quinta tarea del proceso de la dinámica variante.

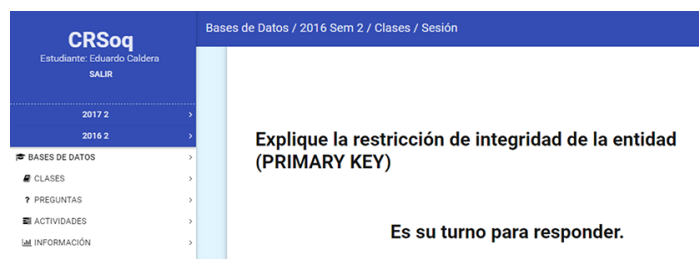

Figura 7. Aviso de turno para responder.

\section{f. Evaluación y finalización de la pregunta}

Posteriormente el profesor debe realizar la sexta tarea del proceso, la que consiste en realizar la evaluación de la respuesta del estudiante, como se aprecia en la Figura 8.

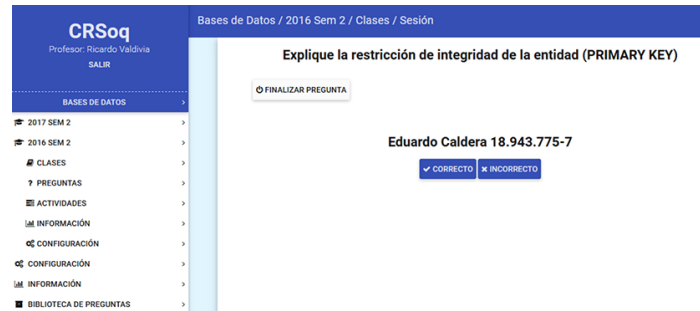

Figura 8. Evaluación de la respuesta del estudiante.

Esta evaluación debe estar basada en criterios propios del profesor y el sistema no interviene ni apoya este aspecto del proceso.

Si la respuesta del estudiante se considera incorrecta, el profesor puede seleccionar a otro estudiante de la lista de participantes (Figura 6). En caso de que la respuesta sea correcta el profesor puede continuar con otra pregunta o finalizar la sesión.

Finalmente, el sistema notifica a cada estudiante del resultado de su evaluación.

\section{Soporte de la Dinámica en el Semestre}

Durante el semestre, el profesor y los estudiantes tienen acceso a información asociada al estado 
de la dinámica, de forma que puedan ajustar su participación en la misma.

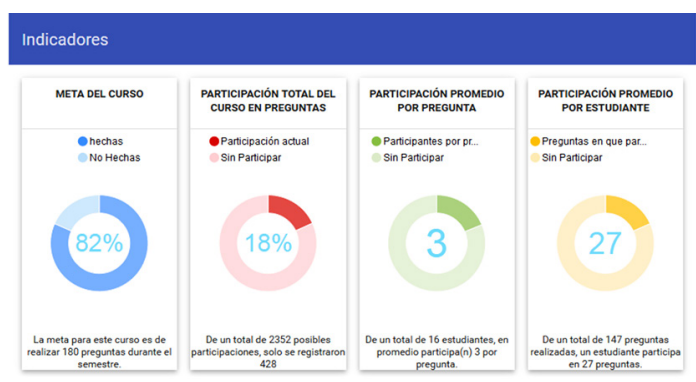

Figura 9. Indicadores de la dinámica.

Los indicadores de la Figura 9 describen cuatro aspectos generales del curso. El primer indicador muestra el grado de avance de la meta del curso. El segundo indicador muestra el grado de participación del curso. El tercer indicador muestra cual es el promedio de estudiantes que participan por pregunta. Finalmente el cuarto indicador señala cual es el promedio de participación de un estudiante.

En la Figura 10 se observa un gráfico que permite visualizar el número de preguntas realizadas clase a clase, ya sea si se respondieron de forma correcta, incorrecta o no tuvieron respuesta.

El detalle de cada día puede visualizarse con detalle al situar el cursor sobre una fecha en particular.

En la Figura 11 se observa un gráfico que indica la cantidad de preguntas realizadas clase a clase versus el nivel de participación.

$\mathrm{Al}$ situar el cursor en una fecha en particular es posible visualizar el porcentaje de participación en la clase.

Para que el profesor pueda evaluar a cada estudiante, es necesario que conozca la cantidad de puntos que ha obtenido en la dinámica. En el gráfico de la Figura 12 el profesor puede visualizar dicha información.

En la Figura 13 se presenta un gráfico con la curva de participación de cada estudiante, necesario para qué el profesor analice el desempeño de cada estudiante clase a clase.

En el gráfico es posible filtrar las curvas de participación por estudiante, de forma de mejorar la visualización de una participación en particular.

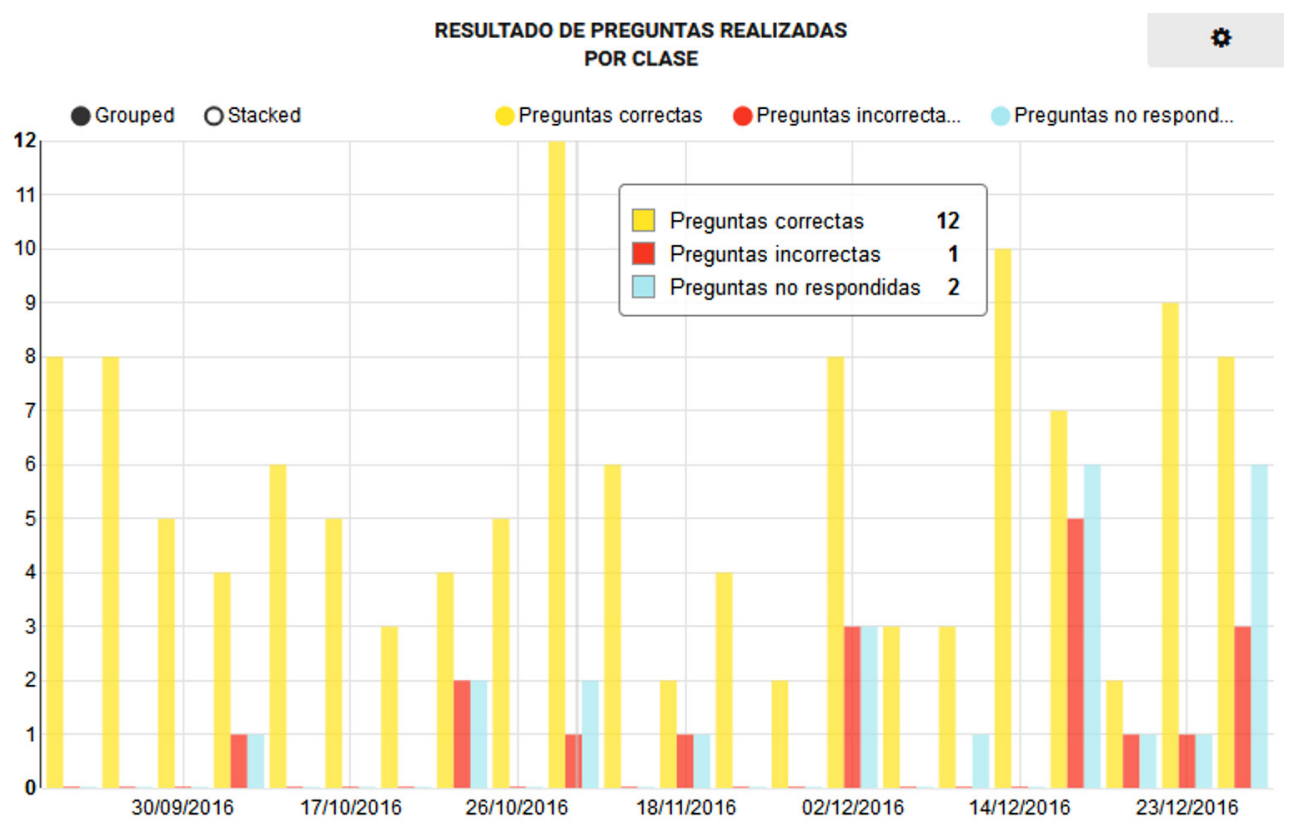

Figura 10. Gráfico de preguntas realizadas clase a clase. 


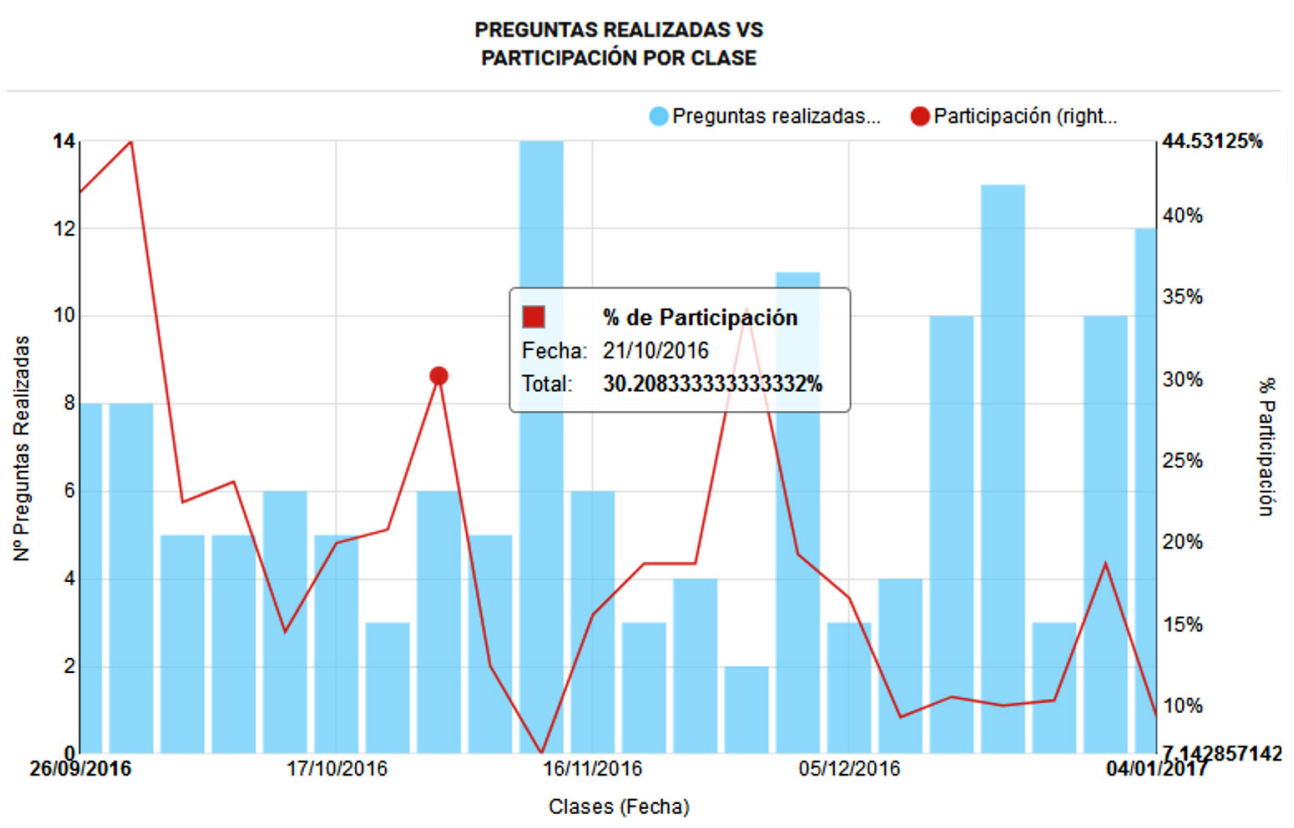

Figura 11. Gráfico de preguntas realizadas versus la participación por clases.

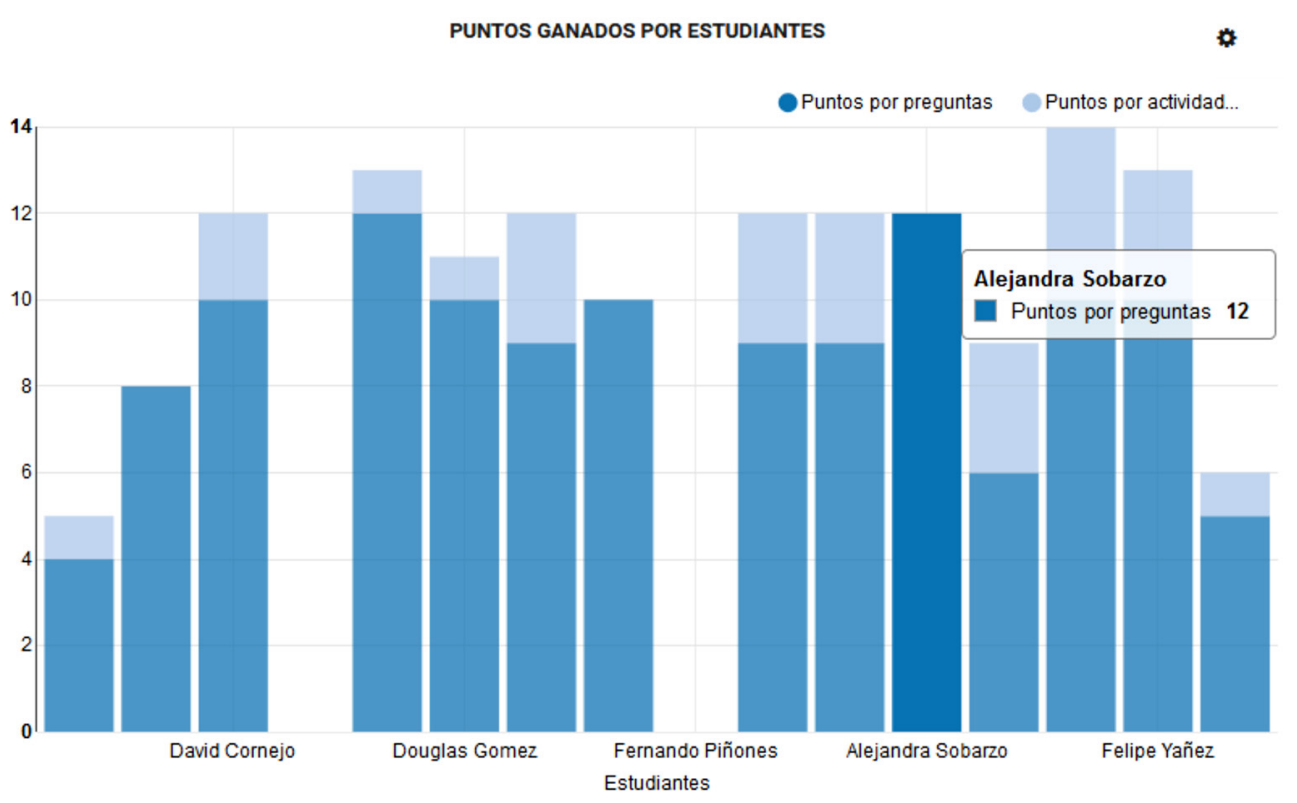

Figura 12. Gráfico de puntos ganados por los estudiantes.

Finalmente, el gráfico de la Figura 14 muestra una estadística por pregunta, con la cual es posible apreciar cómo se produce la actividad al realizar una pregunta en la clase. En el caso mostrado la pregunta fue: ¿Qué es un modelo?, había 17 estudiantes presentes, 6 de los cuales decidieron participar, 4 no fueron seleccionados, 1 respondió en forma incorrecta y 1 correctamente. 


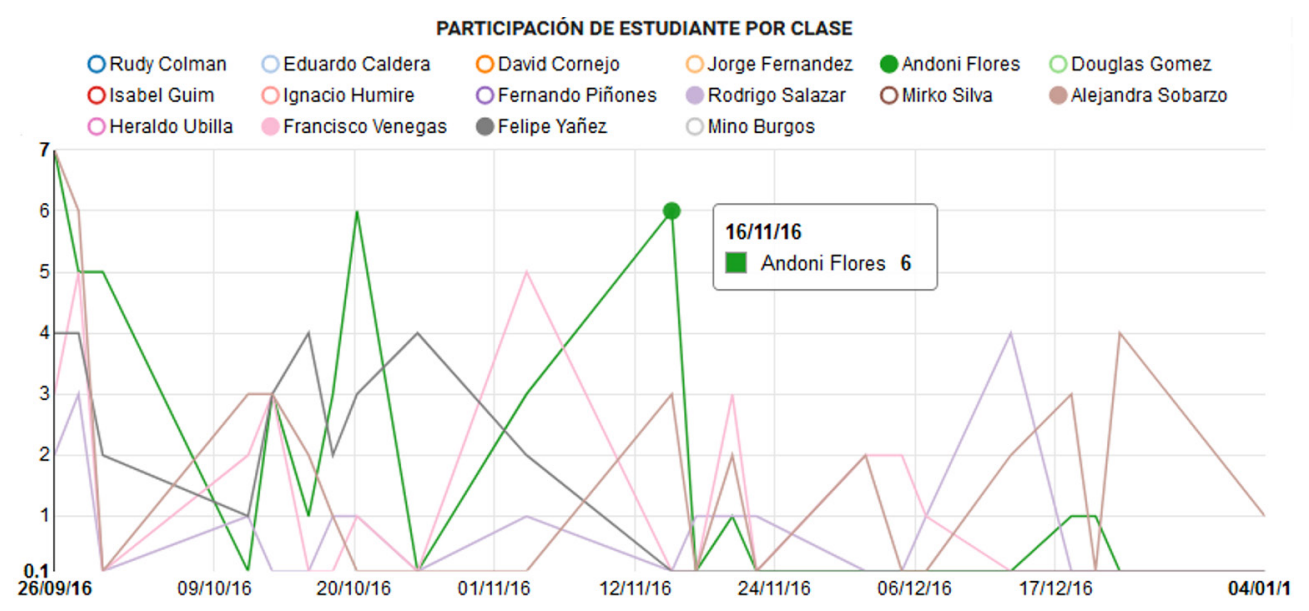

Figura 13. Gráfico de participación por cada estudiante clase a clase.

PARTICIPACIÓN POR PREGUNTA

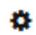

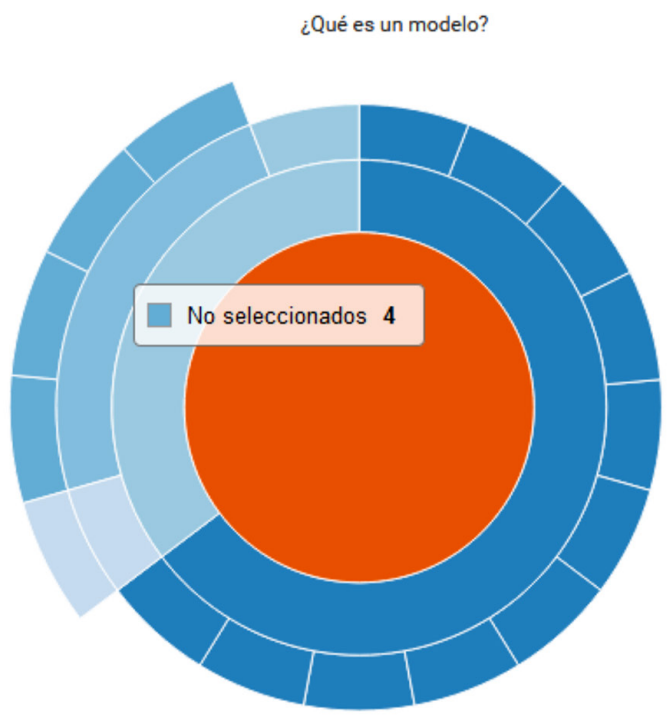

Figura 14. Gráfico de detalle por pregunta.

\section{RESULTADOS}

La dinámica se aplicó en un curso regular de Bases de Datos de una carrera de Ingeniería en Computación e Informática, el segundo semestre de 2016.

El curso estuvo conformado por quince estudiantes, 13 hombres y 2 mujeres, de los cuales 10 realizaban el curso en primera oportunidad. La meta para el semestre se estableció en 180 preguntas, lo que implicaba que cada estudiante debía responder 12 preguntas para obtener la máxima calificación. El porcentaje de aprobación del curso fue de un $80 \%$, y el de la dinámica de un $87 \%$. Al finalizar el curso se había realizado un total de 146 preguntas, lo que representó un $81 \%$ de la meta propuesta.

Al finalizar el curso, se realizó una encuesta de satisfacción de 21 preguntas con una escala de Likert de 5 niveles. La Tabla 2 muestra las 3 preguntas en 
que los estudiantes se mostraron más de acuerdo, las tres preguntas en que los estudiantes se mostraron con mayor desacuerdo y tres preguntas de control con una connotación negativa.

De acuerdo a los resultados obtenidos, los estudiantes manifestaron una actitud positiva, tanto con respecto al uso de la dinámica como hacia el soporte de la herramienta tecnológica. Las preguntas de connotación negativa reafirmaron esta opinión, ya que no se manifestó un rechazo hacia el uso de la dinámica. Las preguntas que obtuvieron una menor aceptación, son aquellas que se refieren a un impacto en el trabajo en equipo o de colaboración entre pares, una propiedad que no estaba considerada en la implementación de la dinámica.

Tabla 2. Encuesta de Satisfacción.

\begin{tabular}{|l|c|c|}
\hline \multicolumn{1}{|c|}{ Preguntas } & $\bar{x}$ & $\mathbf{S}$ \\
\hline $\begin{array}{l}\text { La dinámica soportada por CRSoq ha } \\
\text { contribuido significativamente al desarrollo } \\
\text { de las habilidades prácticas que se requieren } \\
\text { en el curso. }\end{array}$ & 4.5 & 0.65 \\
$\begin{array}{l}\text { La dinámica soportada por CRSoq ha } \\
\text { facilitado mi participación en clases. }\end{array}$ & 4.5 & 0.85 \\
$\begin{array}{l}\text { El nivel de complejidad de las preguntas } \\
\text { realizadas durante el semestre fue el adecuado } \\
\text { para soportar correctamente la dinámica } \\
\text { soportada por CRSoq. }\end{array}$ & 4.9 & 0.36 \\
\hline $\begin{array}{l}\text { La dinámica soportada por CRSoq ha mejorado } \\
\text { mi aprendizaje gracias a la contribución de } \\
\text { mis pares. }\end{array}$ & 39 & 0.95 \\
$\begin{array}{l}\text { La dinámica soportada por CRSoq ha aportado } \\
\text { a mi trabajo efectivo con otros estudiantes. }\end{array}$ & 3.4 & 0.93 \\
$\begin{array}{l}\text { La dinámica soportada por CRSoq ha } \\
\text { contribuido a mejorar mi comunicación con } \\
\text { otros estudiantes. }\end{array}$ & 3.4 & 1.00 \\
\hline $\begin{array}{l}\text { La dinámica soportada por CRSoq no ha } \\
\text { contribuido a desarrollar las competencias } \\
\text { que se persiguen con este curso. } \\
\text { No tomaría otro curso que utilice esta } \\
\text { herramienta tecnológica. } \\
\text { No recomendaría un curso con el uso de esta } \\
\text { herramienta tecnológica a otros estudiantes. }\end{array}$ & 1.5 & 0.65 \\
\hline
\end{tabular}

La Tabla 3 muestra una comparativa con la versión 2015 del curso (conformado por 14 estudiantes) considerando los indicadores promedio final (en escala de 1 a 7) y porcentaje de aprobación.
Tabla 3. Comparación cursos 2015-2016.

\begin{tabular}{|c|c|c|}
\hline Curso & $\bar{x}$ & Aprobación \\
\hline 2015 & 3,9 & $64 \%$ \\
\hline 2016 & 4,5 & $80 \%$ \\
\hline
\end{tabular}

Considerando el indicador promedio final, el $d_{\text {cohen }}$ es de un 0,59 , lo que expresa un efecto medio de la intervención. El cambio producido en el porcentaje de aprobación es destacable, mostrando una variación positiva de un $16 \%$.

\section{CONCLUSIONES}

El uso de la dinámica propuesta permite que los estudiantes expresen sus argumentos al profesor $\mathrm{y}$ al resto de la clase, mejorando sus habilidades expositivas, de pensamiento crítico y reflexivo. El profesor, por su parte, interviene para reforzar y/o corregir los conceptos que han sido expuestos.

El sistema CRSoq está pensado como un sistema de soporte, que presta ayuda al profesor, permitiéndole tomar buenas decisiones en el desarrollo de la dinámica. Esto, a través de la visualización de los niveles de participación y rendimiento de sus estudiantes.

Con el uso del sistema propuesto, el profesor no debe esperar hasta la corrección de las evaluaciones para retroalimentarse del nivel en que se encuentran sus estudiantes. Esto le permite contar con la información necesaria para ir corrigiendo ideas o conceptos. Adicionalmente, el profesor puede ver cómo funciona una pregunta en particular en la historia del curso, permitiéndole ajustar o descartar aquellas que no funcionan como es esperado.

Finalmente, los estudiantes realizaron una valoración global positiva de la aplicación de la dinámica en el curso. Esto se vio reflejado en las siguientes preguntas de la encuesta: "La dinámica soportada por CRSoq ha contribuido significativamente a mi aprendizaje de los contenidos del curso" ( $(\bar{x}=4.4, s=0.65)$, " $L a$ dinámica soportada por CRSoq ha contribuido significativamente al desarrollo de habilidades prácticas que se requieren" $(\bar{x}=4.5, s=0.65)$.

La buena valoración del uso de la dinámica para apoyar el aprendizaje de los contenidos del curso 
puede explicarse en que el uso de las preguntas permitió detectar los aspectos teóricos en que los estudiantes mostraban una mayor debilidad y que, por lo tanto, fueron reforzados. La buena valoración de los aspectos prácticos muestra la flexibilidad en el uso de la herramienta, ya que muchas preguntas fueron asociadas a resultados que los estudiantes debían obtener al realizar una experiencia en clases de laboratorio y que, al ser seleccionados, debían explicar al resto de la clase.

\section{REFERENCIAS}

[1] J.J. Mc Connell. Active Learning and its use in Computer Science, Proceedings of the SIGCSE/SIGCUE Conference on Integrating Technology into Computer Science Education (Barcelona, Spain, June 2-5, 1996) also published as SIGCSE Bulletin, vol. 28, Special Issue, pp. 52-54, 1996.

[2] K.D. Powers \& D.T. Powers. Making Sense of Teaching Methods in Computing Education, FIE'99: Frontiers in Education Conference, San Juan, Puerto Rico, November 1999.

[3] T. Roberts. The Use of Multiple Choice Test for Formative and Summative Assessment, Eighth Australasian Computing Education Conference (ACE2006), Hobart, Tasmania, Australia, January 2006. Conferences in Research in Practice in Information Technology, Vol. 52. Denise Tolhurst and Samuel Mann Eds., 2006.

[4] R.J. Dufresne, W.J. Gerace, W.J. Leonard, J.P. Mestre \& L. Wenk. Classtalk: A Classroom Communication System for Active Learning, Journal of Computing in Higher Education, 7, 3-47, 1996.

[5] E. Mazur, Peer Instruction: A User's Manual. Englewood Cliffs, NJ: Prentice Hall, 1997.
[6] K. Woodford and P. Bancroft. Using Multiple Choice Questions Effectively in Information Technology Education. In R. Atkinson, C. McBeath, D. Jonas-Dwyer \& R. Phillips (Eds.), Beyond the Comfort Zone. Proceedings of the $21^{\text {st }}$ ASCILITE Conference, pp. 948-955, 2004.

[7] J. Roschelle. Unlocking the Learning Value of Wireless Mobile Devices, Journal of Computer Assisted Learning, 19(3), 260272, 2003.

[8] I. Beatty. Transforming Student Learning with Classroom Communication Systems, EDUCAUSE Center for Applied Research, Research Bulletin, Volume 2004, Issue 3, February 3, 2004.

[9] Y. Miao, S. Holst, T. Holmer, J. Freschutz and P. Zentel. An Activity-Oriented Approach to Visually Structured Knowledge Representation for Problem-Based Learning in Virtual Learning Environments, Designing Cooperative Systems. The Use of Theories and Models. Proceedings of the 5th International Conference on the Design of Cooperative Systems (COOP'2000). May 23-26, 2000, Sophia Antipolis, France, pp. 303-318, Frontiers in Artificial Intelligence and Applications. Vol. 58, ISSN 0922-6389, Amsterdam, the Netherlands, IOS Press, 2000.

[10] Object Management Group, "BPMN Specification - Business Process Model and Notation", 2016. [En línea]. Available: http:// www.bpmn.org/. [Último acceso: Junio 2016].

[11] E. Pintor, P. Gargantilla, B. Herreros, M. López, Kahoot en docencia. Una Alternativa Práctica a los Clickers, XI Jornadas Internacionales de Innovación Universitaria: Educar para Transformar, Julio 2014. 